\title{
Crossover between entropic and interfacial elasticity and osmotic pressure in uniform disordered emulsions
}

\begin{abstract}
Thomas G. Mason ${ }^{\star a b}$ and Frank Scheffold ${ }^{c}$
We develop a simple predictive model of the osmotic pressure $\Pi$ and linear shear elastic modulus $G_{p}^{\prime}$ of uniform disordered emulsions that includes energetic contributions from entropy and interfacial deformation. This model yields a smooth crossover between an entropically dominated $G_{p}^{\prime} \sim k_{\mathrm{B}} T / a^{3}$ for droplet volume fractions $\phi$ below a jamming threshold for spheres, $\phi_{\mathrm{C}}$, and an interfacially dominated $G_{p}^{\prime} \sim \sigma / a$ for $\phi$ above $\phi_{c}$, where $a$ and $\sigma$ are the undeformed radius and interfacial tension, respectively, of a droplet and $T$ is the temperature. We show that this model reduces to the known $\phi$-dependent jamming behavior $G_{p}^{\prime}(\phi) \sim(\sigma / a) \phi\left(\phi-\phi_{c}\right)$ as $T \rightarrow 0$ for $\phi>\phi_{c}$ of disordered uniform emulsions, and it also produces the known divergence for disordered hard spheres $G_{p}^{\prime}(\phi) \sim\left(k_{\mathrm{B}} T / a^{3}\right) \phi /\left(\phi_{\mathrm{c}}-\phi\right)$ for $\phi<\phi_{\mathrm{c}}$ when $\sigma \rightarrow \infty$. We compare predictions of this model to data for disordered uniform microscale emulsion droplets, corrected for electrostatic repulsions. The smooth crossover captures the observed trends in $G_{p}^{\prime}$ and $\Pi$ below $\phi_{c}$ better than existing analytic models of disordered emulsions, which do not make predictions below $\phi_{c}$. Moreover, the model predicts that entropic contributions to the shear modulus can become more significant for nanoemulsions as compared to microscale emulsions.
\end{abstract}

\section{Introduction}

Emulsions are among the most versatile and interesting colloidal systems in the realm of dispersed soft matter. ${ }^{1-3} \mathrm{~A}$ strong and rapid osmotic compression of a dilute, monodisperse, uniform emulsion, initially composed of repulsive spherical droplets, yields a disordered jammed colloidal system of deformed droplets. ${ }^{4,5}$ Because the droplets have the capacity to deform and change shape when concentrated, yet the droplet volume is conserved, uniform emulsions are a model system that has provided an excellent starting point for understanding the behavior of disordered soft colloidal glasses that are comprised of compressible building blocks, such as microgel particles, that do not strictly conserve volume when jammed.

Measurements of the mechanical shear moduli of disordered polydisperse emulsions at high concentrations ${ }^{6}$ have been followed by systematic studies of microscale $e^{5,7,8}$ and nanoscale ${ }^{9,10}$ uniform, fractionated, disordered, oil-in-water emulsions as a function of droplet radius $a$ and volume fraction $\phi$. After making minor corrections for electrostatic Debyescreened repulsions between the droplets, these measurements

${ }^{a}$ Department of Physics and Astronomy, University of California-Los Angeles, Los Angeles, CA 90095, USA.E-mail: mason@physics.ucla.edu

${ }^{b}$ Department of Chemistry and Biochemistry, University of California-Los Angeles, Los Angeles, CA 90095, USA

'Department of Physics, University of Fribourg, Chemin du Musée 3, 1700 Fribourg, Switzerland. E-mail: Frank.Scheffold@unifr.ch have clearly linked the dramatic onset of shear elasticity and rise in osmotic pressure to random close packing (RCP) of spheres, $\phi_{\mathrm{RCP}} \approx 0.64$, which over time has been re-defined in terms of the more general language of jamming; ${ }^{11}$ maximal random jamming (MRJ) of disordered monodisperse spheres has been shown in simulations to also occur at $\phi_{\mathrm{MRJ}} \approx 0.64 .^{12,13}$ Regardless of the choice of terminology, there is a critical volume fraction $\phi_{\mathrm{c}} \approx 0.64$, associated with random jamming or close packing of uniform spheres, above which microscale and larger emulsions are typically observed to have osmotic pressures and elastic shear moduli that are readily measurable. It has long been known that non-spherical objects tend to jam and pack at higher critical volume fractions than those associated with spheres. For instance, the classic Kelvin structure of maximally close-packed truncated octahedra, which is a model for "dry" foams and highly compressed emulsions, has a higher packing volume fraction than hexagonally close-packed spheres. More recently, the change in $\phi_{\mathrm{c}}$ as a function of aspect ratio has been measured for disordered, jammed ellipsoids, ${ }^{\mathbf{1 4}}$ again revealing that deformation from a sphere into an ellipsoid increases the volume fraction associated with random jamming.

The measured plateau elastic shear modulus $G_{\mathrm{p}}^{\prime}(\phi)$ and osmotic pressure $\Pi(\phi)$ of uniform microscale emulsions have provided a foundation for developing models, involving both analytic and numerical approaches, that have largely explained the observed trends at high $\phi$ when droplet deformation becomes significant. ${ }^{5,12}$ In addition to identifying soft spots and 
non-affine motion of droplet centers in disordered emulsions that are sheared, Lacasse et al. ${ }^{5,12}$ established a $\phi$-dependent effective spring constant based on Surface Evolver ${ }^{15}$ calculations of droplets having different numbers of contacts. These simulations closely matched the experimental results, which could be captured by simple scaling formulae: $G_{\mathrm{p}}^{\prime}(\phi) \sim(\sigma / a) \phi\left(\phi-\phi_{\mathrm{c}}\right)$ and $\Pi(\phi) \sim(\sigma / a) \phi^{2}\left(\phi-\phi_{\mathrm{c}}\right)$, where $\sigma / a$ represents the Laplace pressure scale and $\sigma$ is the oil-water interfacial tension of the droplets. ${ }^{5,8,16}$ By contrast to $G_{\mathrm{p}}^{\prime}(\phi)$, which follows the above scaling trend for $\phi \geq 0.8$ into the limit of a compressed biliquid foam, $\Pi(\phi)$ rises above the near- $\phi_{\mathrm{c}}$ prediction and diverges as $\phi$ approaches unity. ${ }^{5}$

Later jamming simulations, which rely upon similar computational methods, ${ }^{13,17,18}$ have established a $\phi$-dependent average droplet coordination number near and above $\phi_{\mathrm{c}}$, motivating recent confocal microscopy measurements. ${ }^{19}$ By combining the simulated $\phi$-dependent coordination number ${ }^{17}$ with the $\phi$-dependent effective spring constant ${ }^{12}$ in a more satisfying and self-consistent microscopic description, new analytical formulae have been recently developed for $G_{\mathrm{p}}^{\prime}(\phi)$ and also the yield shear stress $\tau_{\mathrm{y}}(\phi) .{ }^{20}$ The new scaling expression for the shear modulus, $G_{\mathrm{p}}^{\prime} \sim(\sigma / a)\left[\phi^{8 / 3}\left(\phi-\phi_{\mathrm{c}}\right)^{0.82}+\phi^{5 / 3}\left(\phi-\phi_{\mathrm{c}}\right)^{1.82}\right]$ also matches the same measurements and simulations of the elastic moduli of jammed, elastic, disordered spheres., ${ }^{\mathbf{5 1 2}, 20}$ As we have previously shown, ${ }^{20}$ for $\phi \leq 0.8$, this more complex, yet microscopically accurate, expression for the plateau modulus agrees with the simpler original scaling form $G_{\mathrm{p}}^{\prime}(\phi) \sim(\sigma / a) \phi\left(\phi-\phi_{\mathrm{c}}\right)$ to within about $10 \%$ over $\phi_{\mathrm{c}} \leq \phi \leq 0.8$, the entire range of expected validity when the degree of droplet compression is relatively small. So, in cases where analytical simplicity is desired and convenient, the original scaling form for $G_{\mathrm{p}}^{\prime}(\phi)$ can still be used without introducing substantial errors.

By contrast, for charge-stabilized nanoemulsions, electrostatic repulsions between the droplets, even when Debyescreened,,$^{1,2}$ play a major role in the rheology and osmotic equation of state. For instance, the elastic onset of nanoemulsions having $a<100 \mathrm{~nm}$ has been shown to occur at volume fractions near or below $\phi \approx 0.3,{ }^{10}$ far below $\phi_{\mathrm{MRJ}}$. In fact, because the electrostatic contributions are so strong, macroscopic rheology measurements of the $\phi$-dependent plateau elastic shear modulus $G_{\mathrm{p}}^{\prime}(\phi)$ of uniform nanoemulsions can actually be used to measure the Debyescreened potential of interaction $U(h)$, where $h$ is the average closest distance between the interfaces of nearest neighboring droplets, yielding the Debye screening length $\lambda_{\mathrm{D}}$ and the surface potential $\psi_{0} \cdot{ }^{10}$ This approach has been applied in reverse to explain $G_{\mathrm{p}}^{\prime}(\phi)$ of nanoemulsions and also microscale emulsions when the parameters of the Debye-screened potential are known or can be obtained by fitting. ${ }^{21}$ This overall approach of accounting for both droplet deformation and electrostatic repulsions has provided satisfying fits of the rheology of charge-stabilized nanoemulsions over a very wide range of $\phi$.

Here, for colloidal emulsions, to model $\Pi(\phi)$ and $G_{\mathrm{p}}^{\prime}(\phi)$ of disordered uniform droplets over a range of $\phi$ that extends below and above $\phi_{\mathrm{c}}$, we consider both the entropic $^{16,22}$ and interfacial deformation contributions of droplets to the free energy; we neglect electrostatic interactions. Since colloidal droplets diffusively translate in three dimensions at a temperature $T$, the entropic contributions to the free energy $F$ and to $\Pi$ and $G_{\mathrm{p}}^{\prime}$ dominate below $\phi_{c}$, whereas interfacial contributions dominate above it. Such a combined model must effectively capture the entropic $\Pi$ and $G_{\mathrm{p}}^{\prime}$ of glassy hard spheres for $\phi$ below $\phi_{\mathrm{c}}$ in the limit as $\sigma \rightarrow \infty$. A large entropic osmotic pressure $\Pi^{\text {hs }}$ exists for hard spheres near but below $\phi_{c}$; as $\phi$ approaches $\phi_{\mathrm{c}}$, this $\Pi^{\mathrm{hs}}(\phi)$ and $G_{\mathrm{p}}^{\prime}(\phi)$ diverge in a known manner for disordered systems. ${ }^{23}$ Thus far, there is no analytical model that captures the crossover behavior for disordered emulsions both above and below $\phi_{\mathrm{c}}$.

We hypothesize that disordered emulsion droplets at higher osmotic pressures, near the random jamming limit, deform slightly and become non-spherical, as they locally interact and collide, when thermally excited, with nearest neighboring droplets. This time- and ensemble-averaged droplet deformation at local contact points, which is a result of a free energy minimization approach, effectively shifts the divergence point of the entropic term in the free energy. Slight volume-preserving droplet deformations, which increase a droplet's surface area at a cost dictated by the surface tension, create more accessible translational microstates in which a droplet can move before encountering nearest-neighboring droplets. In this simple model, we neglect hydrodynamic interactions and gravitational buoyancy effects, and thus restrict our attention to $\Pi$ and $G_{\mathrm{p}}^{\prime}$ of dense systems of colloidal droplets. Moreover, we assume that the droplet surfaces are uncharged (i.e. $\psi_{0}=0$ ) or, if they are charged, that the Debye screening length $\lambda_{\mathrm{D}}$ is extremely small (i.e. $\lambda_{\mathrm{D}} \rightarrow 0$ ), so that electrostatic contributions to the free energy can be neglected.

The resulting simple model of concentrated disordered uniform emulsions near the jamming point is based on an assumption of quasi-equilibrium thermodynamics, including soft interfacial deformations arising from the disordered structure and the known scaling for the translational free energy of hard spheres in a free energy. By quasi-equilibrium, we mean that the ensemble of most probable, disordered, jammed droplet configurations effectively create average droplet interactions that can be treated by a simple equilibrium free energy minimization approach, even as we recognize that the particular disordered jammed structure is formally an outof-equilibrium state. By writing a free energy, we implicitly assume that the average droplet structure and dynamics do not significantly evolve over experimental time scales used to probe the emulsion's $\Pi$ and $G_{\mathrm{p}}^{\prime}{ }^{16}$ as has been explored more recently for other systems such as disks. ${ }^{24}$ By minimizing a time- and ensemble-averaged local free energy per droplet, we derive analytical formulae for $\Pi(\phi)$ and $G_{\mathrm{p}}^{\prime}(\phi)$ that show a smooth crossover from the glassy entropic scale having energy density $k_{\mathrm{B}} T / a^{3}$ to the Laplace scale having energy density $\sigma / a$. We show that the resulting model captures measurements of the moduli and osmotic pressure both above and below $\phi_{\mathrm{c}}$, in crossover region from the disordered glassy regime to the disordered jamming regime. 


\section{Model}

It is well known that certain hard, three-dimensional, facetted shapes can be packed in ordered lattices to fill space completely; $\phi_{\mathrm{c}}$ for such systems is unity. Hard spheres that are hexagonally close-packed (HCP) into an ordered array have a lower $\phi_{\mathrm{c}}=\phi_{\mathrm{HCP}}$ $\approx 0.74$. Moreover, hard spheres that are randomly jammed (or randomly close packed) into a disordered structure, have an even smaller critical volume fraction at $\phi_{\mathrm{c}}=\phi_{\mathrm{MRJ}}=\phi_{\mathrm{RCP}} \approx$ 0.64. If one takes a randomly jammed structure of spheres and then deforms each sphere slightly without changing its internal volume just at the points where it makes contact with neighboring spheres, one could create tiny facet-like regions, having locally smaller curvatures, on each sphere just where it previously made contact with its nearest neighboring spheres. Such slight deformations would necessarily cause an upward shift in $\phi_{\mathrm{c}}$ to a new, larger, effective critical jamming volume fraction $\phi_{\mathrm{c}}^{\prime}>\phi_{\mathrm{c}}$ of the system of near-spherical particles. A deformation volume fraction, $\phi_{\mathrm{d}}$, can be associated with this shift in the jamming point: $\phi_{\mathrm{d}}=\phi_{\mathrm{c}}^{\prime}-\phi_{\mathrm{c}}$. Likewise, such slight deformations would increase the surface area of each particle, so the average surface area of the near-spherical particles would be larger than for completely spherical particles.

For such a disordered system of hard, near-spherical particles, created in the manner described above, the entropic osmotic equation of state $\Pi(\phi)$ would diverge at the higher $\phi_{\mathrm{c}}^{\prime}$, and not at $\phi_{\mathrm{c}}$. Thus, based on scaling results known for both for ordered and disordered systems of spheres, we infer that the effective free volume available per particle for translational motion would be proportional to $\phi_{\mathrm{c}}^{\prime}-\phi=\phi_{\mathrm{c}}+\phi_{\mathrm{d}}-\phi$. The entropic translational free energy per near-spherical hard particle would be $F_{\text {ent }} / N \sim-k_{\mathrm{B}} T \ln \left(\phi_{\mathrm{c}}^{\prime}-\phi\right) \sim-k_{\mathrm{B}} T \ln \left(\phi_{\mathrm{c}}+\phi_{\mathrm{d}}-\phi\right)$, where $N$ is the number of droplets, and the osmotic pressure of the system of near-spherical hard particles would behave according to $\Pi(\phi) \sim k_{\mathrm{B}} T \phi^{2} /\left[a^{3}\left(\phi_{\mathrm{c}}+\phi_{\mathrm{d}}-\phi\right)\right]$. In free volume models of dense, hard objects, a perturbative shear deformation alters the positional configurations of the particles slightly and effectively reduces the translational free volume fraction by a term proportional to $\alpha \gamma^{2}$, where $\gamma$ is the shear strain and $\alpha$ is a dimensionless parameter, so $F_{\text {ent }} / N \sim-k_{\mathrm{B}} T \ln \left(\phi_{\mathrm{c}}+\phi_{\mathrm{d}}-\phi-\alpha \gamma^{2}\right)$. A linear term in $\gamma$ would be inappropriate, because the free energy must not depend on the particular direction chosen for the shear excitation; using $\alpha \gamma^{2}$ ensures that the free energy reduces to a harmonic function at small $\gamma$. The plateau elastic shear modulus $G_{\mathrm{p}}^{\prime}$ is then calculated by taking a second derivative of $F_{\text {ent }} / N$ with respect to strain and evaluating in the zero strain limit, yielding $G_{\mathrm{p}}^{\prime} \sim k_{\mathrm{B}} T \phi /\left[a^{3}\left(\phi_{\mathrm{c}}+\phi_{\mathrm{d}}-\phi\right)\right]$.

A concentrated system of disordered droplets, which are nearly-spherical but slightly deformed, has $\phi$-dependent distributions of the number of nearest neighboring droplets and of the local deformations (i.e. "facets" near contact points) where a given droplet interacts strongly with its neighbors. For $\phi>\phi_{\mathrm{c}}$, the static interfacial interaction at a given contact between droplets can be anharmonic and even depend on the coordiation number (i.e. the number of other contacts with neighboring droplets). ${ }^{5,12}$ To simplify, in the limit of weak droplet deformations, we assume that all of these effects can be adequately represented by a time- and ensemble-averaged local deformation distance per droplet, $\Delta r$, which lies along a direction normal to the lower curvature regions where nearest neighboring droplet interfaces most closely approach (i.e. normal to flatter "facets") and which is much smaller than the original undeformed droplet radius, $a$. For small $\Delta r$, we assume that $\phi_{\mathrm{d}}$ is linearly proportional to $\Delta r$, so that the gain in accessible translational microstates permitted by the slight deformation is effectively only normal to a local facet. Because the structure of the emulsion is disordered, in the limit of very large numbers of droplets and facets, there is a uniform distribution of these normal directions over the unit sphere. In a perfect crystal of droplets, one would not necessarily expect a linear relationship $\phi_{\mathrm{d}} \sim \Delta r$, so the contact disorder, which leads to this uniform distribution of normal directions at the contact points, as well as the softness that is inherent in the randomly jammed configuration that is less efficiently packed, in the disordered emulsion are important. Simultaneously, we assume that the time- and ensemble-average surface area per deformed droplet, $A_{\mathrm{drop}} / N$, can be represented by an expansion in powers of $\phi_{\mathrm{d}} \sim \Delta r$ as $A_{\mathrm{drop}} / N=4 \pi a^{2}\left(1+\xi \phi_{\mathrm{d}}{ }^{2}+\ldots\right)$, where $\xi$ is a dimensionless geometrical parameter averaged over the entire disordered system and includes a range of differently sized facets and different coordination numbers of the distribution of all droplets. For a volume-preserving deformation of a spherical droplet, we assume that the leading-order surviving term for the increase in surface area per droplet confined is quadratic in the compressional volume change (i.e. in $\phi_{\mathrm{d}}$ and therefore also $\Delta r$ ). Consequently, ignoring the original surface area of the deformed droplets, which is a constant that can be neglected, the interfacial free energy per droplet associated with weak droplet deformation in the disordered emulsion, on average, is simply $F_{\text {int }} / N=4 \pi \sigma a^{2} \xi \phi_{\mathrm{d}}^{2}$.

We propose that the osmotic pressure and linear shear modulus of a collection of disordered uniform droplets near $\phi_{\mathrm{c}}$ that are entropically excited can be obtained by minimizing the total free energy per droplet, $F / N=F_{\text {int }} / N+F_{\text {ent }} / N$, with respect to the degree of droplet deformation, as captured by $\phi_{\mathrm{d}}$. Although energy minimization is an equilibrium approach, we apply it to the disordered system of droplets, recognizing that the particular configurational state is disordered and not strictly in equilibrium. In doing so, we implicitly assume that, despite the vast number of different possible out-of-equilibrium configurations of disordered droplets, the average over the most common configurations in a disordered emulsion (i.e. corresponding to maximal random jamming) can be expressed using a simple free energy per droplet. We define $V$ to be the total volume of the emulsion and $V_{\text {drop }}=4 \pi a^{3} / 3$ to be the volume of a droplet. $N$ droplets occupy a system volume $V$ at a temperature $T$. We define the average free volume of accessible translational microstates of the center of mass of a droplet to be $V_{\mathrm{f}}$. The number of accessible states $\Omega$ of the system is approximately the $N$ th power of the ratio of the free volume per droplet to the volume of the unit cell enclosing a droplet, $V_{\mathrm{uc}}: \Omega \sim\left(V_{\mathrm{f}} / V_{\mathrm{uc}}\right)^{N}$. To estimate the entropic contribution, we consider deformed droplets to effectively pack at $\phi_{\mathrm{c}}^{\prime}$ but to exhibit entropic scaling 
characteristic of hard spheres. At a high density the center of each droplet can only move in a free volume within a cage of neighboring spheres that is proportional to the product of the volume per sphere with the cube of the volume fraction difference away from jamming: ${ }^{23} V_{\mathrm{f}} \sim V_{\mathrm{uc}}\left(\phi_{\mathrm{c}}^{\prime}-\phi\right) .{ }^{3}$ According to Boltzmann's law, the translational entropy in three dimensions is then: $S=k_{\mathrm{B}} \ln \Omega=k_{\mathrm{B}} \ln \left(\phi_{\mathrm{c}}^{\prime}-\phi\right)^{3 N}=3 N k_{\mathrm{B}} \ln \left(\phi_{\mathrm{c}}^{\prime}-\phi\right)$. The entropic contribution to the free energy is $F_{\text {ent }} / N=-T S / N$, so the total free energy per droplet is:

$$
F / N=4 \pi \sigma a^{2} \xi \phi_{\mathrm{d}}^{2}-3 k_{\mathrm{B}} T \ln \left(\phi_{\mathrm{c}}+\phi_{\mathrm{d}}-\phi\right) .
$$

The increase in effective area per droplet $\sim \phi_{\mathrm{d}}{ }^{2}$ in the first term represents an average over a complex distribution of interfacial areas of deformation that depends on the average number and proximity of nearest neighbors as well as the specific nature of the deformations of droplets in the disordered emulsion. Because of this "soft deformation" assumption, we would expect any consequent relationships to be valid near and above $\phi_{\mathrm{c}}$ (i.e. in the approximate range $0.55 \leq \phi \leq 0.80$ ), yet well below unity where the osmotic pressure of an emulsion diverges.

In summary, neglecting electrostatic repulsions, the free energy for droplets near close packing contains two contributions: an energy associated with the translational motion of the droplets in a restricted cage made up of neighboring droplets and an energy associated with the deformation of the droplets themselves. The free volume available for translational motion near jamming is affected by the degree of droplet deformation, which effectively shifts the jamming point away from that of perfect spheres, and yet work must be done to overcome interfacial tension in order for the deformation to occur, in a selfconsistent manner. Droplets that nearly touch may deform a slight amount on average near their contact points to make more microstates for translation accessible at the expense of creating a slightly higher surface area, as illustrated for a single contact between two droplets in Fig. 1(a) and (b). This tradeoff can be formally expressed by minimizing the total free energy, the sum of entropic and interfacial terms, with respect to the degree of droplet deformation. Incorporating both interfacial and entropic energetic contributions (yet neglecting the constant undeformed droplet area), yields the free energy per droplet as a function of droplet volume fraction and shear strain amplitude:

$$
F(\phi, \gamma) / N=4 \pi a^{2} \sigma \xi \phi_{\mathrm{d}}^{2}-3 k_{\mathrm{B}} T \ln \left(\phi_{\mathrm{c}}+\phi_{\mathrm{d}}-\phi-\alpha \gamma^{2}\right) .
$$

In principle, this simple model could be refined using more sophisticated microscopic expressions for the free energy, but such expressions would not necessarily result in a much more accurate connection between the entropic and interfacial scales of energy density than eqn (2), which is more convenient analytically. We emphasize that the model that we have developed implicitly assumes that droplets are already trapped in cages of neighboring droplets formed at $\phi$ below $\phi_{c}$. We make no attempt to describe the caging mechanism related to the

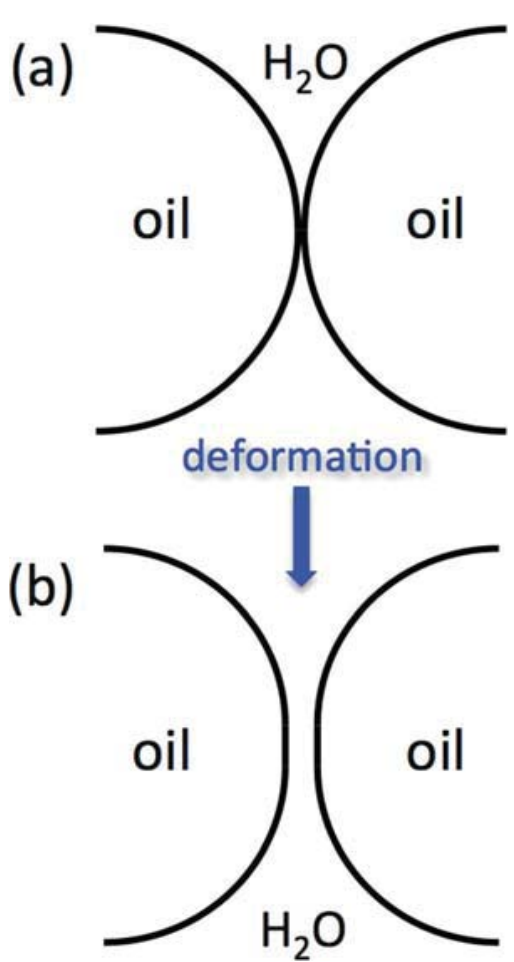

Fig. 1 Schematic diagram of entropic deformation of a contact between two oil droplets in a continuous aqueous liquid at a positive non-zero temperature. (a) Packed droplets that touch cannot translate; for hard spheres, the osmotic pressure and shear moduli diverge at the jamming point, given by the volume fraction $\phi_{\mathrm{c}} \approx 0.64$. (b) Slight deformations of the droplets in the vicinity of contacts create additional accessible translational microstates for neighboring droplets; this is entropically favorable.

origin of the entropic elasticity that emerges as a consequence of the glass transition some percentile below $\phi_{\mathrm{c}}{ }^{25}$

We link the translational free volume to the droplet deformation by minimizing the free energy with respect to $\phi_{\mathrm{d}}$, so that the droplets deform a small amount in order to increase the number of translational accessible microstates:

$$
\left.\frac{\partial F}{\partial \phi_{\mathrm{d}}}\right|_{\phi_{\mathrm{d}}=\phi_{\mathrm{d}}^{*}}=0,
$$

which determines $\phi_{\mathrm{d}}^{*}$ in terms of $\phi, \phi_{\mathrm{c}}$, and $\gamma$ : This equation effectively expresses the balance of the translational entropic pressure of deformed droplets with the increased interfacial pressure due to their average deformation. Applying the minimization condition to eqn (2), we find:

$$
8 \pi \xi \frac{\sigma}{a} \phi_{\mathrm{d}}^{*}-\frac{3 k_{\mathrm{B}} T}{a^{3}}\left[\frac{1}{\phi_{\mathrm{c}}+\phi_{\mathrm{d}}^{*}-\phi-\alpha \gamma^{2}}\right]=0 .
$$

Solving for $\phi_{\mathrm{d}}^{*}$ using the quadratic equation, one finds the positive, physical root:

$$
\phi_{\mathrm{d}}^{*}=\frac{1}{2}\left\{\left[\phi-\left(\phi_{\mathrm{c}}-\alpha \gamma^{2}\right)\right]+\sqrt{\left[\phi-\left(\phi_{\mathrm{c}}-\alpha \gamma^{2}\right)\right]^{2}+\phi_{\mathrm{T}}^{2}}\right\},
$$

where we define $\phi_{\mathrm{T}}$ : 


$$
\phi_{\mathrm{T}}^{2} \equiv\left(\frac{3 k_{\mathrm{B}} T}{a^{3}}\right) /\left(2 \pi \xi \frac{\sigma}{a}\right),
$$

as the square of a dimensionless volume fraction representing the ratio of the entropic energy density scale $\sim k_{\mathrm{B}} T / a^{3}$ to an interfacial energy density scale $\sim \sigma / a$. The minimized $\phi_{\mathrm{d}}^{*}$ in eqn (5) can be substituted back into the free energy in eqn (2) to obtain an explicit dependence of the quasi-equilibrium minimal free energy on $\phi$ and $\gamma$.

To obtain the osmotic pressure $\Pi$ and plateau shear modulus $G_{\mathrm{p}}^{\prime}$, we simply calculate appropriate thermodynamic derivatives of this free energy subject to the minimization condition. In a quiescent state without any applied shear strain, we set $\gamma=0$, take the thermodynamic derivative of the free energy density with respect to $\phi$ and calculate $\Pi=\left[\phi^{2} /\left(N V_{\text {drop }}\right)\right] \partial F / \partial \phi:$

$$
\Pi(\phi)=\frac{3 k_{\mathrm{B}} T}{V_{\mathrm{drop}}}\left[\frac{\phi^{2}}{\phi_{\mathrm{c}}+\phi_{\mathrm{d}}^{*}-\phi}\right],
$$

noting that in the total derivative of $F$ with respect to $\phi$, when subject to the condition of energy minimization in eqn (3), only the partial derivative that is explicitly with respect to $\phi$ survives. Using the minimization condition in eqn (4), we next convert eqn (7) from the entropic scale to the interfacial energy scale. Substituting for $\phi_{\mathrm{d}}^{*}$ from eqn (5) in the absence of shear when $\gamma=0$, we alternatively determine $\Pi$ in terms of the Laplace pressure scale and $\phi_{\mathrm{T}}$ :

$$
\Pi(\phi)=3 \xi \frac{\sigma}{a} \phi^{2}\left[\left(\phi-\phi_{\mathrm{c}}\right)+\sqrt{\left(\phi-\phi_{\mathrm{c}}\right)^{2}+{\phi_{\mathrm{T}}}^{2}}\right],
$$

which reduces to $\Pi \sim(\sigma / a) \phi^{2}\left(\phi-\phi_{\mathrm{c}}\right)$ as $T$ approaches zero Kelvin, i.e. as $\phi_{\mathrm{T}}$ approaches 0. Eqn (8) is valid for disordered soft spheres for $\phi$ near but below $\phi_{\mathrm{c}}$ and also above $\phi_{\mathrm{c}}$ in the weak deformation jamming regime but not in the limit of significant droplet deformation for $\phi \geq 0.80$. The osmotic compressional modulus $K_{\Pi}$ can be calculated by taking an appropriate thermodynamic derivative of $\Pi(\phi)$ with respect to $\phi,{ }^{16}$ again subject to the energy minimization condition. The resulting formula for the osmotic compressional modulus rises more abruptly near $\phi_{\mathrm{c}}$ than the osmotic pressure.

Next, using the free energy at the minimization condition, we calculate the plateau shear modulus $G_{\mathrm{p}}^{\prime}=\left[\phi /\left(N V_{\mathrm{drop}}\right)\right] \partial^{2} F /\left.\partial \gamma^{2}\right|_{\phi_{\mathrm{d}}=\phi_{\mathrm{d}}^{*}, \gamma=0}$ retaining all terms after differentiating, before evaluating at $\gamma=0$. The result is:

$$
\begin{gathered}
G_{\mathrm{p}}^{\prime}(\phi)=\frac{6 \alpha k_{\mathrm{B}} T}{V_{\mathrm{drop}}}\left[\frac{\phi}{\phi_{\mathrm{c}}+\phi_{\mathrm{d}}^{*}-\phi}\right] \\
=6 \alpha \xi \frac{\sigma}{a} \phi\left[\left(\phi-\phi_{\mathrm{c}}\right)+\sqrt{\left(\phi-\phi_{\mathrm{c}}\right)^{2}+\phi_{\mathrm{T}}^{2}}\right] .
\end{gathered}
$$

When entropy becomes unimportant (i.e. as $T \rightarrow 0$ and $\phi_{\mathrm{T}} \rightarrow 0$ ), weak deformation of disordered droplets dominates for $\phi \geq \phi_{\mathrm{c}}$, so eqn (10) reduces to $G_{\mathrm{p}}^{\prime}=12 \alpha \xi(\sigma / a) \phi\left(\phi-\phi_{\mathrm{c}}\right)$. This result essentially matches the scaling expression $G_{\mathrm{p}}^{\prime} \sim(\sigma / a) \phi\left(\phi-\phi_{\mathrm{c}}\right)$ that has been used to fit both experimental data ${ }^{5}$ and simulation results ${ }^{12}$ well for $\phi_{\mathrm{c}} \approx 0.64<\phi<0.80$.
In addition to the $T \rightarrow 0$ jamming limit of the model's predictions for $\Pi$ and $G_{\mathrm{p}}^{\prime}$, evaluated above, there is another interesting limiting case: the dense limit of disordered hard spheres for $\sigma \rightarrow \infty$ when $\phi<\phi_{\mathrm{c}}{ }^{2,22,26}$ In this limit, the minimum deformation parameter for $\phi<\phi_{\mathrm{c}}$ vanishes: $\phi_{\mathrm{d}}^{*} \approx\left[\left(\phi-\phi_{\mathrm{c}}\right)+\left|\phi-\phi_{\mathrm{c}}\right|\right] / 2 \rightarrow 0$. Consequently, the hard-sphere osmotic pressure reduces to $\Pi^{\mathrm{hs}}(\phi)=3 k_{\mathrm{B}} T \phi^{2} /\left[V_{\mathrm{drop}}\left(\phi_{\mathrm{c}}-\phi\right)\right]$ for $\phi \leq \phi_{\mathrm{c}}$. Likewise, the shear modulus reduces to the known prediction for hard spheres: $G_{\mathrm{p}}^{\prime} \mathrm{hs}(\phi)=6 \alpha k_{\mathrm{B}} T \phi /\left[V_{\mathrm{drop}}\left(\phi_{\mathrm{c}}-\phi\right)\right]$. The proportionality of $\Pi^{\mathrm{hs}}$ and $G_{\mathrm{p}}^{\prime} \mathrm{hs}$ to $k_{\mathrm{B}} T / a^{3}$ and their divergence with $\left(\phi_{\mathrm{c}}-\phi\right)^{-1}$ are consistent with simulations for dense hard sphere systems. ${ }^{2,23}$ The simplification of eqn (8) and (10) to the known jamming limit for emulsions and also to the known divergence behavior of hard spheres also confirms that $F_{\text {int }} \sim \phi_{\mathrm{d}}^{2}$ is appropriate for describing the average interfacial work done against interfacial tension in disordered emulsions.

\section{Results and discussion}

As a test of the model, in Fig. 2, using eqn (10), we fit measurements of the scaled $G_{\mathrm{p}}^{\prime}\left(\phi_{\text {eff }}\right) /(\sigma / a)$ for a fractionated microscale silicone oil-in-water emulsion having $a=0.53 \mu \mathrm{m}$, where the electrostatic repulsion has largely been taken into account through the effective volume fraction $\phi_{\text {eff. }}$ For this emulsion, which is stabilized by anionic surfactant sodium dodecyl sulfate (SDS), the interfacial tension is $\sigma=9.8$ dyn $\mathrm{cm}^{-1}$. The temperature is $T=298 \mathrm{~K}$. The shear strain amplitude is $\gamma \approx 0.01$, corresponding to the upper limit of the linear regime, yet still maximizing the torque on the rheometer, which is important as measured values of $G_{\mathrm{p}}^{\prime}$ decrease many orders of

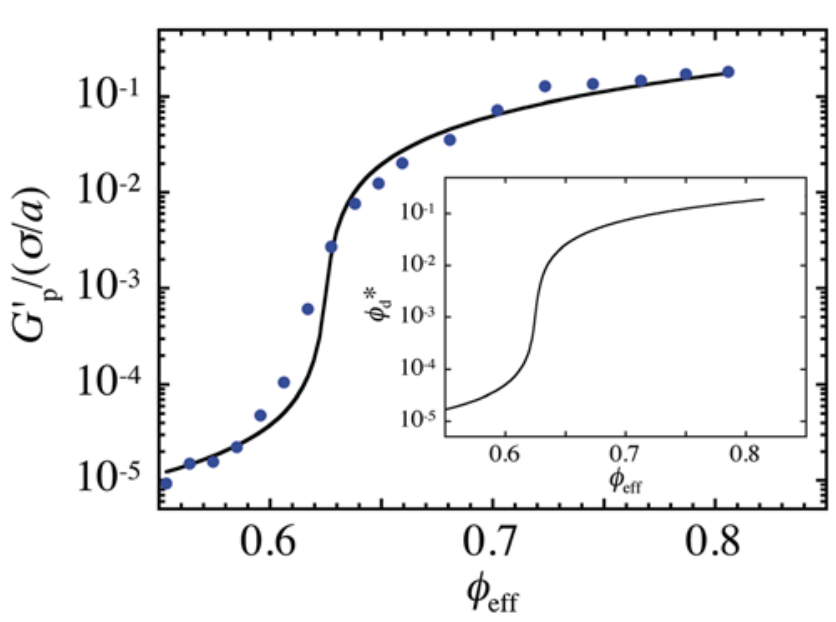

Fig. 2 Measured plateau elastic shear modulus $G_{p}^{\prime}$ divided by the Laplace pressure scale $\sigma / a$ as a function of effective volume fraction $\phi_{\text {eff }}$ for a uniform silicone oil-in-water emulsion having average radius $a=0.53 \mu \mathrm{m}$ and oil-water interfacial tension $\sigma=9.8 \mathrm{dyn} \mathrm{cm}^{-1}$. Solid line is a fit to the measurement using eqn (10), yielding fit parameter values of $\phi_{\mathrm{c}}=0.625 \pm 0.005, \xi=0.14 \pm 0.06$, and $\alpha=0.74 \pm 0.32$. The model captures the smooth crossover near $\phi_{c}$ between the entropic and Laplace scales of energy density inherent in the measured shear modulus. Data shown are from ref. 5 (used with permission). Inset: the deformation volume fraction $\phi_{\mathrm{d}}^{*}$ resulting from free energy minimization using eqn (5) and (6) and the fit parameter values above. 
magnitude. We allow three parameters to vary in the fit: $\alpha, \xi$, and $\phi_{c}$, and the fit is weighted so that points below $\phi_{c}$ approaching the entropic scale are important. The parameters determined from the fits are: $\phi_{\mathrm{c}}=0.625 \pm 0.005, \xi=0.14 \pm$ 0.06 , and $\alpha=0.74 \pm 0.32$. The fit value of $\phi_{\mathrm{c}}$ is very close to $\phi_{\mathrm{MRJ}} \approx 0.64$ and represents good agreement, given the uncertainties in the experimental measurements of the reported values of $\phi$ and the subsequent correction of this $\phi$ for the Debye-screened repulsion to obtain $\phi_{\text {eff }}$. The dimensionless interfacial-entropic coupling parameter $\xi$ and the shearcoupling parameter $\alpha$ have values near but below unity. These parameters depend on microscopic features related to the average geometry of a droplet encaged by its neighbors in the disordered emulsion, and an exact theoretical determination of the values of these parameters are not known. The fit captures the smooth crossover between the entropic energy density scale and the interfacial scale. There is a slight systematic deviation of the data from the fit near $\phi_{c}$, and this deviation could arise from a number of sources: inadequate corrections of the electrostatic interactions inherent in $\phi_{\text {eff }}$, not explicitly including $\phi$-dependent coordination numbers and spring constants in the model, and residual droplet polydispersity (which in the experimental emulsion is between about $10-15 \%$ ), which will tend to smear the entropic and Laplace pressure scales and thereby affect the transition region near $\phi_{\mathrm{c}}$. In the inset of Fig. 2, we also calculate the deformation volume fraction $\phi_{\mathrm{d}}^{*}$, corresponding to the minimal free energy, using values of the fit parameters in eqn (5) and (6). For any given $\phi$, whether above or below the jamming limit, the sum given by $\phi_{\mathrm{c}}+\phi_{\mathrm{d}}^{*}$ is always greater than $\phi$. Moreover, $\phi_{\mathrm{d}}^{*}(\phi)$ increases significantly in the vicinity of $\phi=\phi_{\mathrm{c}}$, where the disordered droplets begin to jam and deform, on average. The model captures these effects using system-averaged parameters and free energy minimization, even as shear excitations can cause non-affine positional configurational displacements of individual droplets because of the disorder.

Using the parameters obtained from the fit of $G_{\mathrm{p}}^{\prime}(\phi)$ at $a=$ $0.53 \mu \mathrm{m}$, which reflects measured shear moduli over a large dynamic range for a real, uniform, disordered, oil-in-water emulsion, we predict the crossover in the elastic moduli using eqn (10) for droplet radii $a=5 \mathrm{~nm}, 50 \mathrm{~nm}, 500 \mathrm{~nm}$, and $5 \mu \mathrm{m}$, where $\sigma=10$ dyn $\mathrm{cm}^{-1}$ and $T=300 \mathrm{~K}$, as shown in Fig. 3. For 5 $\mu \mathrm{m}$ and larger droplets, beyond the colloidal scale, the entropic energy density is so far below the Laplace scale that it would be very difficult to detect and hence can be neglected in most practical situations. While difficult to measure, entropic contributions to the plateau shear modulus of microscale droplets having $a=500 \mathrm{~nm}$ are detectable, yet even in this range, the plateau modulus is measured at an intermediate frequency, and does not necessary reflect a zero-frequency modulus. For larger nanoemulsions having $a=50 \mathrm{~nm}$, the entropic energy density is still substantially below the Laplace scale, but for smaller nanoemulsions having $a=5 \mathrm{~nm}$, the entropic contribution becomes quite important and can significantly modify the overall magnitude and shape of $G_{\mathrm{p}}^{\prime}(\phi)$ (see inset of Fig. 3). For real oil-in-water nanoemulsions stabilized using SDS, the screened charge repulsions are known to

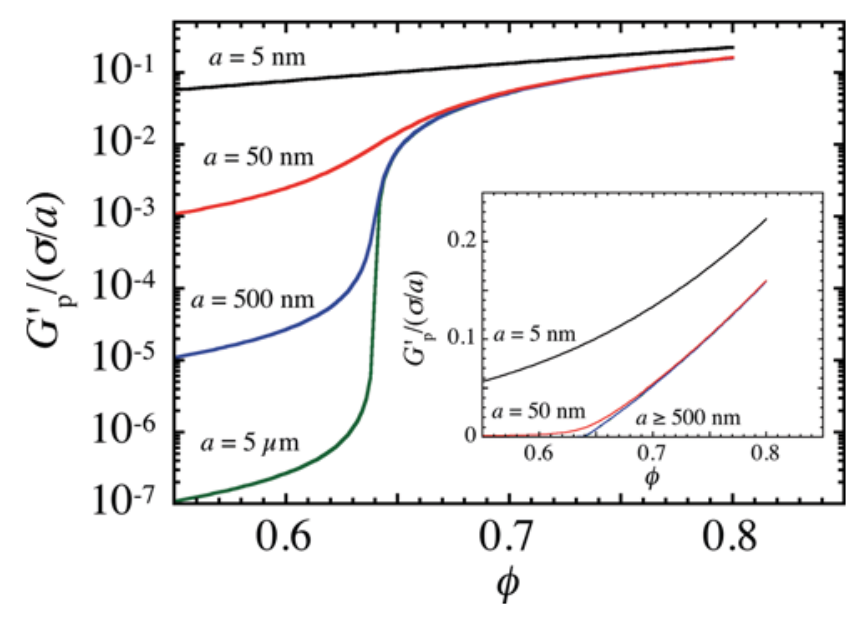

Fig. 3 Predictions of the smoothly varying crossover regime of the elastic shear modulus $G_{p}^{\prime}$ for droplet volume fractions $\phi$ just above and below the jamming point $\phi_{\mathrm{c}} \approx 0.64$ for soft deformable near-spherical emulsion droplets for $T=298 \mathrm{~K}$ and $\sigma=9.8 \mathrm{dyn} \mathrm{cm}^{-1}$, according to eqn (10): lines (from top to bottom) radii $a=5 \mathrm{~nm}, 50 \mathrm{~nm}, 500 \mathrm{~nm}$, and $5 \mu \mathrm{m}$. Values of $\xi$ and $\alpha$ from the fit in Fig. 2 are used. Inset: linear-linear format showing a prediction that very fine nanoemulsions could significantly deviate from $\phi\left(\phi-\phi_{c}\right)$ behavior near the jamming point.

have a much larger effect than for microscale emulsions, and their moduli can be explained by models that incorporate only interfacial deformations and screened charge. ${ }^{\mathbf{1 0 , 2 1}}$ However, the predictions we have made herein might be useful for non-ionic nanoemulsions in which screened charge repulsions are absent. Recent simulations of soft disordered spheres ${ }^{17}$ appear to give a crossover very similar to the analytic eqn (10), which predicts scaling and crossover aspects between the entropic and the interfacial energy densities in concentrated disordered emulsion systems.

The measured osmotic equation of state $\Pi\left(\phi_{\text {eff }}\right) /(\sigma / a)$ of a similar uniform disordered microscale oil-in-water emulsion having average $a=0.48 \mu \mathrm{m}$ and stabilized using SDS at the same conditions is shown in Fig. 4. Because it is difficult to measure very low osmotic pressures of these emulsions directly, there is significant scatter in the data near and below $\phi_{\mathrm{c}}$. Using eqn (8), we fit the measured $\Pi\left(\phi_{\text {eff }}\right)$, allowing $\xi$ and $\phi_{\mathrm{c}}$ to vary as parameters, and we obtain $\xi=0.16 \pm 0.06$ and $\phi_{\mathrm{c}}=0.62 \pm 0.01$. Within the uncertainties, these values of fit parameters obtained from $\Pi\left(\phi_{\text {eff }}\right)$ overlap well with the fit parameters obtained from the measured $G_{\mathrm{p}}^{\prime}\left(\phi_{\text {eff }}\right)$. For reference, in the inset of Fig. 4, we show predictions of the osmotic pressure in the crossover region for macroscale, microscale, and nanoscale emulsions. Again, for macroscale droplets beyond the colloidal scale, the entropic contribution to the osmotic pressure can be neglected. For microscale droplets, the entropic contribution is difficult to detect using conventional means, but begins to become discernable. Because $\left(k_{\mathrm{B}} T / a^{3}\right) /(\sigma / a) \sim a^{-2}$, which is apparent for both $\Pi$ and $G_{\mathrm{p}}^{\prime}$ when $\phi$ is well below $\phi_{\mathrm{c}}$, for nanoscale droplets, the entropic contribution becomes much more significant; thus, nanoemulsions appear to offer the greatest opportunity for measuring the crossover regime above and below $\phi_{\mathrm{c}}$. 


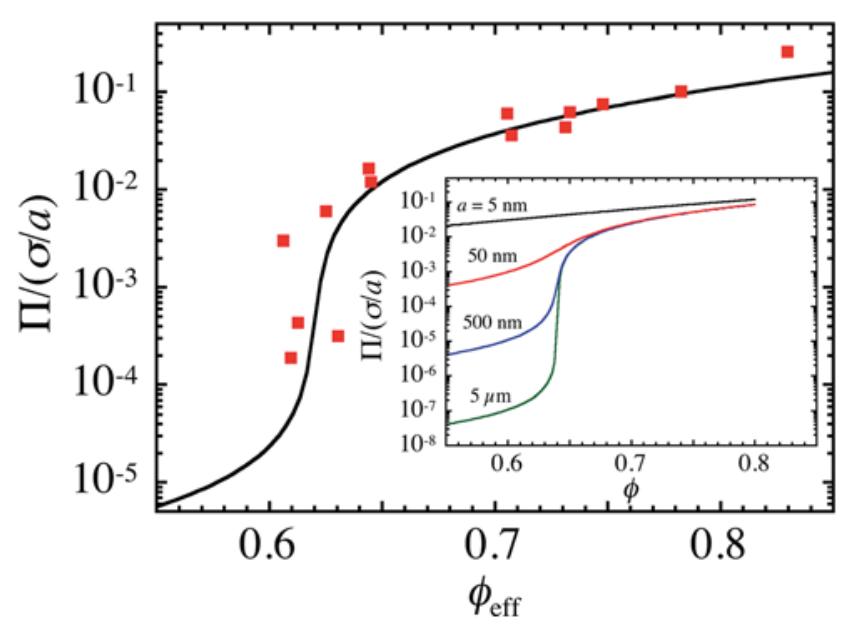

Fig. 4 Measured osmotic equation of state $\Pi\left(\phi_{\text {eff }}\right)$ divided by the Laplace pressure scale $\sigma / a$ of a uniform disordered silicone oil-inwater emulsion having an average radius $a=0.48 \mu \mathrm{m}$. Here, $T=298 \mathrm{~K}$ and $\sigma=9.8 \mathrm{dyn} \mathrm{cm}^{-1}$. The fit is given by eqn (8), and the fit parameters are $\phi_{\mathrm{c}}=0.62 \pm 0.01$ and $\xi=0.16 \pm 0.06$. Data shown are from ref. 5 (used with permission). Inset: predicted smooth crossover in the osmotic equation of state $\Pi(\phi)$ for uniform disordered emulsions having a ranging from $5 \mathrm{~nm}$ to $5 \mu \mathrm{m}$ with $\phi_{\mathrm{c}}=0.64$.

\section{Conclusion}

For concentrated, disordered, uniform, colloidal emulsions, we have used relatively simple thermodynamic arguments based on energy minimization to derive analytic predictions for the smooth crossover of the osmotic pressure and plateau shear modulus of colloidal emulsions from the entropic to the Laplace energy density scales as $\phi$ is increased. The assumption of "soft" deformations of the droplets in the disordered system, in combination with the effective shift in the jamming point accommodated by the deformation, provides a simple yet adequate coupling between the two terms in the free energy, that, when minimized, yields scaling forms for $G_{\mathrm{p}}^{\prime}(\phi)$ and $\Pi(\phi)$ that are consistent with experimental measurements both above $\phi_{\mathrm{c}}$ in the jamming regime and below $\phi_{\mathrm{c}}$ in the entropic glassy regime. Other choices of the assumed scaling of the interfacial and entropic terms with $\phi_{\mathrm{d}}$ prior to minimization would yield other forms of the smooth crossover, but not necessarily provide the known limiting cases for hard spheres and jammed deformable emulsions. This energy minimization approach, modeling ensemble behavior in a disordered system, predicts the observed $G_{\mathrm{p}}^{\prime}(\phi)$ for uniform microscale emulsions near $\phi_{\mathrm{c}}$, thus going beyond pure mechanical analysis that considers only droplet deformation. ${ }^{27}$ Although the model describes the measurements reasonably well over an extended range, a theoretical calculation of the coupling parameters $\xi$ and $\alpha$ in a disordered emulsion system would be useful in order to compare with values obtained from the fits of experimental data. Obviously, the model that we have presented for disordered droplets is not intended to capture or describe strong aging or shear-induced ordering effects, which can be present after subjecting emulsions to a large amplitude pre-shear. ${ }^{28}$
The simple model that we have developed is most directly applicable to non-ionic, quiescent, disordered, colloidal emulsions, in which electrostatic repulsions can be neglected. However, when $\lambda_{\mathrm{D}} \ll a$, we have shown that making minor corrections to $\phi$ through $\phi_{\text {eff }}$ is sufficient to provide a good comparison for charge-stabilized microscale emulsions. In the future, we anticipate that a more comprehensive model of the osmotic equation of state and linear shear rheology of uniform disordered charge-stabilized colloidal emulsions, ranging from the nanoscale to the microscale, can be developed by including a third term in the free energy, which is properly linked to $\phi_{\mathrm{d}}$ through Debye screened electrostatic interactions, and by minimizing this total combined entropic + electrostatic + soft interfacial jamming free energy. Furthermore, by including the more accurate microscopic picture, the predictions of $\Pi(\phi)$ and $G_{\mathrm{p}}^{\prime}(\phi)$ for disordered colloidal emulsions can be refined, and predictions of the yield shear stress $\tau_{\mathrm{y}}(\phi)$ could also be obtained..$^{20}$ Exploring the implications of this model in relation to aging and dynamical heterogeneity effects, ${ }^{25}$ large-amplitude pre-shearing conditions, ${ }^{28}$ frequency-dependent and strain-rate dependent rheology, ${ }^{29}$ attractive interactions, ${ }^{30}$ and polydispersity in the distribution of droplet radii remains on the frontier.

Differences in the local average pair interactions between disordered dispersions of other colloidal objects could create significant differences in their shear rheology and osmotic equation of state in the crossover regime, as compared to emulsions. Droplets in emulsions are just one type of soft object, but they offer the advantage of having a very well defined droplet volume, because they are incompressible, yet still the possibility of soft behavior and effective shifting of the jamming point through deformation. Other soft colloidal systems, such as dispersions of solid spheres stabilized by polymers ${ }^{3,31-34}$ and star polymers, ${ }^{35-37}$ can exhibit different crossover behavior because the local interactions are not the same as between emulsion droplets, and also because these objects are compressible or have compressible shells, making a strict definition of the particle volume fraction difficult. In the future, it will be interesting to compare and contrast the crossover behavior of a wide variety of disordered soft colloidal systems.

\section{Acknowledgements}

T.G.M. thanks P.M. Chaikin, M.-D. Lacasse, D. Levine, J. Bibette, and D.A. Weitz for past discussions. F.S. acknowledges financial support by the Swiss National Science Foundation under grant number 149867.

\section{References}

1 R. G. Larson, The Structure and Rheology of Complex Fluids, Oxford Univ. Press, New York, 1999.

2 W. B. Russel, D. A. Saville and W. R. Schowalter, Colloidal Dispersions, Cambridge Univ. Press, Cambridge, 1989.

3 J. Mewis and N. J. Wagner, Colloidal Suspension Rheology, Cambridge Univ. Press, Cambridge, 2012. 
4 T. G. Mason, Curr. Opin. Colloid Interface Sci., 1999, 4, 231238.

5 T. G. Mason, M.-D. Lacasse, G. S. Grest, D. Levine, J. Bibette and D. A. Weitz, Phys. Rev. E, 1997, 56, 3150-3166.

6 H. M. Princen and A. D. Kiss, J. Colloid Interface Sci., 1986, 112, 427.

7 T. G. Mason, J. Bibette and D. A. Weitz, Phys. Rev. Lett., 1995, 75, 2051-2054.

8 T. G. Mason, A. H. Krall, H. Gang, J. Bibette and D. A. Weitz, in Encyclopedia of Emulsion Technology, ed. P. Becher, Marcel Dekker, New York, 1996, vol. 4.

9 M. M. Fryd and T. G. Mason, Annu. Rev. Phys. Chem., 2012, 63, 493-518.

10 J. N. Wilking and T. G. Mason, Phys. Rev. E, 2007, 75, 041407. 11 M. van Hecke, J. Phys.: Condens. Matter, 2010, 22, 033101.

12 M.-D. Lacasse, G. S. Grest, D. Levine, T. G. Mason and D. A. Weitz, Phys. Rev. Lett., 1996, 76, 3448-3451.

13 S. Torquato, T. M. Truskett and P. G. Debenedetti, Phys. Rev. Lett., 2000, 84, 2064.

14 A. Donev, I. Cisse, D. Sachs, E. A. Variano, F. H. Stillinger, R. Connelly, S. Torquato and P. M. Chaikin, Science, 2004, 303, 990-993.

15 K. A. Brakke, Experiment. Math., 1992, 1, 89-173.

16 T. G. Mason, Rheology of Monodisperse Emulsions, Ph.D. Dissertation, Princeton University, 1995.

17 A. Ikeda, L. Berthier and P. Sollich, Phys. Rev. Lett., 2012, 109, 018301.

18 C. S. O'Hern, L. E. Silbert, A. J. Liu and S. R. Nagel, Phys. Rev. $E, 2003,68,011306$.

19 I. Jorjadze, L.-L. Pontani and J. Brujic, Phys. Rev. Lett., 2013, 110, 048302.

20 F. Scheffold, F. Cardinaux and T. G. Mason, J. Phys.: Condens. Matter, 2013, 25, 502101.
21 F. Scheffold, J. N. Wilking, J. Haberko, F. Cardinaux and T. G. Mason, Soft Matter, 2014, 10, 5040-5044.

22 T. G. Mason and D. A. Weitz, Phys. Rev. Lett., 1995, 75, 27702773.

23 L. V. Woodcock, Ann. N. Y. Acad. Sci., 1981, 371, 274.

24 D. Bi, J. Zhang, B. Chakraborty and R. P. Behringer, Nature, 2011, 480, 355.

25 L. Berthier, G. Biroli, J.-P. Bouchaud, L. Cipelletti and W. van Saarloos, Dynamical Heterogeniety in Glasses, Colloids, and Granular Matter, Oxford Univ. Press, Oxford, 2011.

26 P. Pusey and W. van Megen, Nature, 1986, 320, 340.

27 D. Stamenovic and T. A. Wilson, J. Appl. Mech., 1984, 51, 229.

28 J.-R. Huang and T. G. Mason, Europhys. Lett., 2008, 83, 28004.

29 J. M. Brader, T. Voigtmann, M. Fuchs, R. G. Larson and M. E. Cates, Proc. Natl. Acad. Sci. U. S. A., 2009, 106, 15186.

30 S. S. Datta, D. D. Gerrard, T. S. Rhodes, T. G. Mason and D. A. Weitz, Phys. Rev. E, 2011, 84, 041404.

31 G. Fritz, V. Schädler, N. Willenbacher and N. J. Wagner, Langmuir, 2002, 18, 6381-6390.

32 A. A. Potanin and W. B. Russel, Phys. Rev. E, 1995, 52, 730737.

33 Polymers in Confined Environments, ed. S. Granick, SpringerVerlag, Berlin, 1999.

34 J. Mewis and P. Dhaene, Makromol. Chem., Macromol. Symp., 1993, 68, 213-225.

35 C. N. Likos, H. Löwen, M. Watzlawek, B. Abbas, O. Jucknischke, J. Allgaier and D. Richter, Phys. Rev. Lett., 1998, 80, 4450-4453.

36 G. Foffi, F. Sciortino, P. Tartaglia, E. Zaccarelli, F. Lo Verso, L. Reatto, K. A. Dawson and C. N. Likos, Phys. Rev. Lett., 2003, 90, 238301.

37 D. Vlassopoulos, J. Polym. Sci., Part B: Polym. Phys., 2004, 42, 2931-2941. 